Cite as: Stephane H Maes, (2020), “No Gravity Shield in Multi-folds Universes”, viXra:2010.0032v1, https:// vixra.org/pdf/2010.0032v1.pdf, https://shmaesphysics.wordpress.com/2020/06/26/no-gravity-shields-inmulti-folds-universes/, June 26, 2020.

\title{
No Gravity Shield in Multi-folds Universes
}

\author{
Stephane H. Maes ${ }^{1}$
}

June 24, 2020

\begin{abstract}
:
In a multi-fold universe, gravity emerges from Entanglement through the multi-fold mechanisms. As a result, gravity-like effects appear in between entangled particles that they be real or virtual. Long range, massless gravity results from entanglement of massless virtual particles. Entanglement of massive virtual particles leads to massive gravity contributions at very smalls scales. Multi-folds mechanisms also result into a spacetime that is discrete, with a random walk fractal structure and non-commutative geometry that is Lorentz invariant and where spacetime nodes and particles can be modeled with microscopic black holes. All these recover General relativity at large scales and semi-classical model remain valid till smaller scale than usually expected. Gravity can therefore be added to the Standard Model. This can contribute to resolving several open issues with the Standard Model.
\end{abstract}

The presence of a matter obstacle or shield on the path of the entangled virtual photons may be understood as weakening the gravity perceived beyond or within by a test particle. It is an incorrect conclusion. The potential energy (momentum 4-vector) of the shield and the shield acting a new source ensure that gravity perceived by the test particle is unaffected (other than by the additional contributions due to the proper gravity of the shield).

In a multi-fold universe, Faraday cages do not weaken gravity!

\section{Introduction}

The new preprint [1] proposes contributions to several open problems in physics like the reconciliation of General Relativity with Quantum Physics, explaining the origin of gravity proposed as emerging from quantum (EPREinstein Podolsky Rosen) entanglement between particles [5], detailing contributions to dark matter and dark energy and explaining other Standard Model mysteries without requiring New Physics beyond the Standard Model other than the addition of gravity to the Standard Model Lagrangian. All this is achieved in a multi-fold universe that may well model our real universe, which remains to be validated.

With the proposed model of [1], spacetime and Physics are modeled from Planck scales to quantum and macroscopic scales and semi classical approaches appear valid till very small scales. In [1], it is argued that spacetime is discrete, with a random walk-based fractal structure, fractional and noncommutative at, and above Planck scales (with a 2-D behavior and Lorentz invariance preserved by random walks till the early moments of the universe). Spacetime results from past random walks of particles. Spacetime locations and particles can be modeled as microscopic black holes (Schwarzschild for photons and spacetime coordinates, and metrics between Reisner Nordstrom [2] and Kerr Newman [3] for massive and possibly charged particles - the latter being possibly extremal). Although surprising, [1] recovers results consistent with other like [4], while also being able to justify the initial assumptions of black holes from the gravity or entanglement model. The resulting gravity model recovers General Relativity (GR) at larger scale, as a 4-D process, with massless gravity, but also with massive gravity components at very small scale that make gravity significant these scales. Semi-classical models also work well till way smaller scales than usually expected.

\footnotetext{
${ }^{1}$ shmaes.physics@gmail.com
} 
In the present paper, which is just a short note, we remain at a high level of analysis. It makes the points accessible to a wider audience and keeps the door open to further papers or discussions devoted to details of interest. Yet, it requires the reader to review [1], as we do not revisit here all the details of the multi-fold mechanisms or reconstruction of spacetime. However, we hope that it will intrigue enough the reader to push him or her to dig deeper. Most of the more detailed (or entry point) references are provided in [1], and so every statement is not motivated here or presented with the most appropriate references. This paper is rather a story tale. "[1]" appears often, as a person or a model, to refer to the original arguments, analysis, mechanisms or proposals discussed in [1].

The paper discuss a topic not discussed in [1]: how to deal with matter shielding that path of virtual photons as gravity emerges from the entanglement of virtual particles.

\section{Gravity through or behind a larger mass or inside a Faraday cage}

The mechanisms of multi-folds, the main feature proposed in [1], trigger activation of additional structures (folds) when particles are (EPR) entangled so that additional paths can traverse the folds, where the EPR entangled particles can always meet as a same exit points. Doing so, all the activated folds (i.e. multi-folds) create attractive

potentials in $\frac{1}{r}$ in between the entangled particles $\left(\frac{1}{r^{2}}\right.$ per fold). The attraction is towards their source or center the mass, depending of the use cases and movements (and masses involved - entangled particles can be massive or massless). When involving virtual particles emitted by a source of energy, this potential is reminiscent of gravity and [1] attributes gravity to these effects. It can also be looked as adding contributions of the Ricci curvature scalar R of the folds, from all matter or energy contributions, to build a new Ricci curvature scalar field R and, with the direction of attraction information, a new consistent Ricci curvature tensor. Doing so, for all sources of energy, recovers Einstein's GR field equations (or Hilbert Einstein Action); which is amazing as invariance of surfaces (the real geometrical meaning behind the Hilbert Einstein Action) or variants of the Hilbert Einstein have, at no point, be postulated in [1] prior to that determination (something that can't exactly be said the same way for strings). Also, the multi-folds have a spin-2 symmetry.

Because gravity is explained via the virtual particles in [1] an incorrect but logical expectation would be that behind a large mass or within a cage, especially a massive Faraday cage; gravity in a multi-fold universe would be weakened as at large scale, only neutrinos would go through the shield and carry multi-fold effects. If it were the case the model and mechanisms of a multi-fold universe would not model well our real universe.

Fortunately, such expectation is not correct; gravity is not affected by such obstacles or shields.: there are no gravity shields.

[1] must be able to account for no weakening of gravity within Faraday cages for example, despite our mechanisms relying on virtual particles. If only virtual neutrinos were to contribute, gravity would be weakened within such a cage, which is obviously not the case. In general for the multi-fold mechanisms of [1], when the virtual particles tries to reach a test particle within an electromagnetic shield, it does affect the four -vector potential of the particles on shield. Considering the system shield + target particle, its total energy is affected and it affects the energy source available to multi-folds affecting the test particle. The combined effect is hierarchical and the composition appears as if the effect went through the shield: The mass of the shield particle is increased to reflect the potential from the source. The outcome is the same as if no shield were present except form the additional proper gravitational effect of the shield as source.

\section{Conclusions}


The multi-fold mechanism proposed in [1] Is not defeated by matter shield: gravity is not weakened: instead of only letting neutrinos contribution through the shield adds a contribution that reflects that as a source of energy it is subject to the potential energies from the source; which resolves the perceived issue and maintain suitability of the model of gravity emergence from entanglement of virtual particle emitted by a source of energy (or mass).

References: (most references come from popular science to make the discussion more approachable)

[1]: Stephane H. Maes, (2020) "Quantum Gravity Emergence from Entanglement in a Multi-Fold

Universe", viXra:2006.0088v1, (June 9, 2020).

[2]: https://en.wikipedia.org/wiki/Reissner\%E2\%80\%93Nordstr\%C3\%B6m metric

[3]: https://en.wikipedia.org/wiki/Kerr-Newman metric

[4]: Burinskii, Alexander, (2008), "The Dirac-Kerr-Newman electron", arXiv:0507109v4 